UW-ThPh-2012-17

AEI-2012-36

\title{
Evolution of a periodic eight-black-hole lattice in numerical relativity
}

\author{
Eloisa Bentivegna \\ Max-Planck-Institut für Gravitationsphysik \\ Albert-Einstein-Institut \\ Am Mühlenberg 1, D-14476 Golm \\ Germany
}

\author{
Mikołaj Korzyński \\ Gravitational Physics \\ Faculty of Physics \\ University of Vienna, A-1090 Vienna \\ Austria
}

\begin{abstract}
The idea of black-hole lattices as models for the large-scale structure of the universe has been under scrutiny for several decades, and some of the properties of these systems have been elucidated recently in the context of the problem of cosmological backreaction. The complete, three-dimensional and fully relativistic evolution of these system has, however, never been tackled. We explicitly construct the first of these solutions by numerically integrating Einstein's equation in the case of an eight-black-hole lattice with the topology of $S^{3}$

PACS numbers: 04.25.dg, 04.20.Ex, 98.80.Jk
\end{abstract}

\section{Introduction}

The vast amount of large-scale cosmological data collected in recent decades has shaped a generally coherent picture of our universe [1, where the thermodynamics and nucleosynthesis in the early universe, the generation of the seeds of cosmic structure and their subsequent evolution all fit together in a simple framework based on remarkably few principles. This success, however, comes at the price of accepting the existence of a dark sector, i.e. two fluids, dark matter [2] and dark energy [3, 4], which have rather peculiar physical properties, have an uncertain collocation within the Standard Model, and have never been observed in terrestrial laboratories despite accounting for over $95 \%$ of the energy density of the universe.

Whilst this result could very well point to the existence of new physical constituents or principles, the possibility that the current way we model the inhomogeneous universe be too elementary (a possibility that was advanced for the first time in [5]) has now resurfaced [6. In particular, the question of the extent to which cosmic inhomogeneities can dress the value of the cosmological parameters is 
under scrutiny in a variety of approaches $7,8,9,10,11,12,13$, (see also the review articles [14, 15] and references therein).

An interesting class of models that has been studied for some time is that of regular lattices of black holes [16, 17, These representations of our universe avoid the issues related to the behavior of relativistic fluids (and, in particular, the corresponding singularities); one could argue that they also represent a more realistic picture of our universe, composed of a collection of pointlike objects surrounded by vacuum rather than a homogeneous and isotropic fluid with small-scale perturbations.

Collections of black holes also have the added benefit to be one of numerical relativity's routine application areas [18, from which formalisms, tools and practices can be readily imported. In this work, we construct the initial data and simulate the evolution of a special sort of black hole lattices, those with extrinsic curvature that is initially zero. In section 2, we illustrate how to construct exact initial data for a generic black-hole lattice based on the usual Lichnerowicz-York framework 19, 20. We then discuss a coordinate transformation that simplifies the numerical treatment in section 2.2, illustrate the details of the evolution in section 3 , interpret the results and contrast them to the homogeneous and isotropic class in 4 and finally conclude in section 5. Unless otherwise stated, greek indices run from 0 to 4 , latin indices run from 1 to 3 , and we set $G=c=1$.

\section{Constructing a periodic black-hole lattice}

Given the standard $3+1$ split of the metric tensor into the spatial metric $\gamma_{i j}$ and extrinsic curvature $K_{i j}$, initial data for the gravitational field can be generated by solving the Hamiltonian and momentum constraints, which read:

$$
\begin{aligned}
& R+K^{2}-K_{i j} K^{i j}=16 \pi G \rho \\
& D_{j} K_{i}^{j}-D_{j} K=8 \pi G j_{i}
\end{aligned}
$$

where $R$ is the scalar curvature of the spatial metric and $D_{i}$ represents the covariant derivative associated with $\gamma_{i j} ; \rho=n^{\mu} n^{\nu} T_{\mu \nu}$ and $j^{i}=-\gamma^{i j} n^{\mu} T_{j \mu}$ represent the energy and momentum density respectively.

Let us assume that $j_{i}$ vanishes. A powerful scheme to generate solutions of this system is the conformal transverse-traceless framework, which entails the introduction of a conformal transformation in the spatial metric, along with the separation of the extrinsic curvature into its trace $K$ and traceless part $A_{i j}$ :

$$
\begin{aligned}
& \gamma_{i j}=\psi^{4} \tilde{\gamma}_{i j} \\
& K_{i j}=\frac{K}{3} \gamma_{i j}+A_{i j}
\end{aligned}
$$

In terms of these, the constraints take the form:

$$
\begin{aligned}
& \tilde{\Delta} \psi-\frac{\tilde{R}}{8} \psi-\frac{K^{2}}{12} \psi^{5}+\frac{1}{8} \tilde{A}_{i j} \tilde{A}^{i j} \psi^{-7}=-2 \pi G \psi^{5} \rho \\
& \tilde{D}_{i} \tilde{A}^{i j}-\frac{2}{3} \psi^{6} \tilde{\gamma}^{i j} \tilde{D}_{i} K=0
\end{aligned}
$$

$\ddagger$ Notice that the usual definition of a black hole in an asymptotically-flat spacetime is inapplicable to these spaces. Here, by black hole we denote a spacetime region surrounded by a marginally outer-trapped tube (MOTT). 


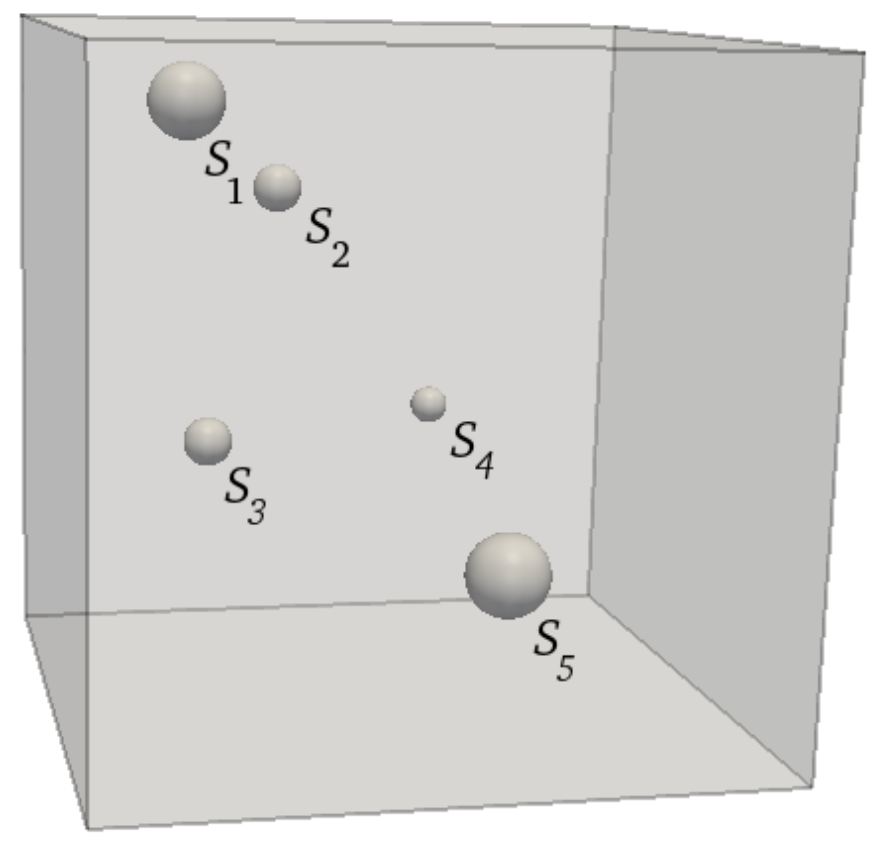

Figure 1. The elementary cell of a periodic 3-space containing a number of punctures and corresponding inner boundaries, e.g. in the shape of spherical surfaces $S_{i}$.

$\tilde{\Delta}$ being the laplacian operator of the conformal metric $\tilde{\gamma}_{i j}$, and $\tilde{A}_{i j}$ being related to $A_{i j}$ by $\tilde{A}_{i j}=\psi^{2} A_{i j}$ 政,

Let us focus on the Hamiltonian constraint. We would like to solve this equation with periodic boundary conditions. We also allow for matter content in form of ordinary matter $\rho$ as well as in "punctures", i.e. singularities in $\psi$ of the form $m_{i} / 2 r$, $m_{i}>0$. It can be easily proven that, unlike in the asymptotically-flat case, if we set both $K_{i j}$ and $R$ to zero, then this is a slice of Minkowski spacetime.

To see this, let us first integrate both sides of equation (5) over the fundamental cell $D$ (which, for the sake of illustration, we will assume cubical) of the desired lattice, with small balls around the punctures excised at the surfaces $S_{i}$ (see Figure 1). The volume integral of $\tilde{\Delta} \psi$ can then be turned into a surface integral on the $S_{i}$ alone, as $\tilde{\Delta} \psi$ identically vanishes on the periodic boundary. We thus obtain:

$$
\int_{D}\left(\frac{\tilde{R}}{8} \psi+\frac{1}{12} K^{2} \psi^{5}-\frac{1}{8} \psi^{-7} \tilde{A}^{i j} \tilde{A}_{i j}\right) \sqrt{\tilde{\gamma}} d^{3} x=
$$

$\S$ A different scaling of $\rho$ is preferable if we want to solve the initial value problem for some types of matter, but this plays no role in our argument. 


$$
=2 \pi G\left(\int_{D} \rho \psi^{5} \sqrt{\tilde{\gamma}} d^{3} x+\Sigma_{i=1}^{N} m_{i}\right) .
$$

On the right hand side, which is manifestly positive, we have the total energy content of the cell both in form of a continuous distribution as well as in the punctures. If we set both $\tilde{R}$ and $K$ to zero, the equation becomes impossible to satisfy unless the matter content vanishes as well. Thus, for non-zero $m_{i}$, we need to admit either a non-zero extrinsic curvature $K$ or a positive spatial scalar curvature $\tilde{R}$.

In this work we concentrate on the second case, setting $K$ to vanish. In this case the momentum constraint is trivally satisfied and the Hamiltonian constraint remains linear in $\psi$, which allows for constructing multiple-black-hole solutions by superposition.

We would like the conformal metric $\tilde{\gamma}$ to be periodic just like the physical one. The simplest way to ensure that it admits a discrete symmetry is to assume that it is a hyperbolic, spherical or flat metric. Since the integral of $\tilde{R}$ must be positive, $\tilde{\gamma}$ must be the metric of a round 3-sphere. This condition limits both the form of the metric tensor and the topology of the lattice $\|$.

Let us note that a similar reasoning applies to the Friedmann-LemaitreRobertson-Walker (FLRW) models: the Hamiltonian constraint in this class reads

$$
\frac{R}{8}+\frac{K^{2}}{12}=2 \pi G \rho
$$

Since the RHS, representing the matter content, is manifestly positive, then either we must have non-vanishing Hubble parameter $K$, or a positive curvature $R$ (or both). Thus, if we assume $K=0$, then there is a similarly strong restriction on the metric tensor and the topology of the constant-time slices.

\subsection{Punctures on $S^{3}$}

Following [21] [1, we consider puncture-like solutions of the Hamiltonian constraint when $\tilde{\gamma}_{i j}$ and $R$ are respectively the metric tensor $\gamma_{i j}^{S}$ and the scalar curvature of the round 3-sphere:

$$
\tilde{\Delta} \psi-\frac{\tilde{R}}{8} \psi=0
$$

We fix coordinates on $S^{3}$ such that:

$$
\gamma^{S}=\mathrm{d} \lambda^{2}+\sin ^{2} \lambda\left(\mathrm{d} \theta^{2}+\sin ^{2} \theta \mathrm{d} \varphi^{2}\right)
$$

Let us imagine that the sphere is embedded in $\mathbf{R}^{4}$ with the equation $\left(X^{1}\right)^{2}+\left(X^{2}\right)^{2}+$ $\left(X^{3}\right)^{2}+\left(X^{4}\right)^{2}=1$; a bar over a capital letter denotes a vector in this space.

Equation (9) has no regular solutions, but it is straightforward to find its solutions with a puncture-type singularity:

$$
\psi(\lambda)=\frac{A}{\sin \lambda / 2} .
$$

|| In a more general setting, the scalar curvature does not have a definite sign and the integral condition (7) gives little information. Nevertheless, thanks to the Yamabe theorem, $\tilde{\gamma}$ must be conformally equivalent to a constant-curvature metric. If we rewrite equation (7) in terms of this metric, it obviously becomes a condition for the sign of the Yamabe energy $\mathcal{E}(\tilde{\gamma})$.

ฯ The analysis of this initial-data construction in the context of the backreaction problem has also recently appeared in 22] 
or in Cartesian coordinates

$$
\begin{aligned}
& \psi(\bar{X})=A \sqrt{\frac{2}{1-\bar{X} \cdot \bar{N}}}, \\
& \bar{N}=(0,0,0,1)
\end{aligned}
$$

We can easily superimpose $N$ such punctures centered at chosen locations $\bar{N}_{i} \in S^{3}$ :

$$
\psi(\bar{X})=\sum_{i=1}^{N} \frac{A_{i}}{\sin \lambda_{i} / 2}=\sum_{i} A_{i} \sqrt{\frac{2}{1-\bar{X} \cdot \bar{N}_{i}}}
$$

The parameters $A_{i}>0$ measure the singular part of the solution at the points $\bar{N}_{i}$ : the leading part behaves like $2 A_{i} / \lambda_{i}$.

Notice that, if one seeks only the regular arrangements of black holes on $S^{3}$, there are only six possible values of $N$, corresponding to the six regular tessellations of the 3-sphere: $N=5,8,16,24,120,600$. In the following, we concentrate on the 8-vertex, 16-cell solution, where the puncture locations are given by:

$$
\begin{aligned}
& \bar{N}_{1}=(1,0,0,0), \\
& \bar{N}_{2}=(-1,0,0,0), \\
& \bar{N}_{3}=(0,1,0,0), \\
& \bar{N}_{4}=(0,-1,0,0), \\
& \bar{N}_{5}=(0,0,1,0), \\
& \bar{N}_{6}=(0,0,-1,0), \\
& \bar{N}_{7}=(0,0,0,1), \\
& \bar{N}_{8}=(0,0,0,-1) .
\end{aligned}
$$

and all $A_{i}=1$. The configuration obviously has the symmetry of a 16-cell. In particular, it has a discrete group of symmetries generated by $\pi / 2$ rotations around all pairs of axes of $\mathbf{R}^{4}$ and reflections about all four hyperplanes perpendicular to the axes. The elementary cell in this pattern is cubical in shape, i.e. it has 6 faces, 8 edges and 8 vertices, at which exactly 4 edges meet. All edges lie on great circles of $S^{3}$ and their length is equal to 168.343 .

\subsection{Stereographic projection of $S^{3}$}

Since it is easier to perform the evolution of asymptotically-flat data as opposed to data on a sphere, we employ the stereographic projection from the top of the sphere into $\mathbf{R}^{3}$, given by

$$
x^{i}=\frac{2 X^{i}}{1-X^{4}}
$$

It is well known that the projection is a conformal mapping in the sense that

$$
\gamma_{i j}^{S}=\left(|\vec{x}|^{2} / 4+1\right)^{-2} \delta_{i j}
$$

or

$$
\gamma_{i j}^{S}=\left(\sin \frac{\lambda}{2}\right)^{-4} \delta_{i j}=\left(\frac{2}{1-\cos \lambda}\right)^{2} \delta_{i j}
$$


where $\delta_{i j}$ is the flat metric. The physical metric 14 projected down to $\mathbf{R}^{3}$ takes the form of

$$
\begin{aligned}
\psi^{4} \gamma_{i j}^{S} & =\left(A_{1}\right)^{4} \tilde{\psi}^{4} \delta_{i j} \\
\tilde{\psi}(\vec{x}) & =1+\sum_{i=2}^{N} \frac{2 A_{i} \sqrt{1+\left|\vec{n}_{i}\right|^{2} / 4}}{A_{1}} \cdot \frac{1}{\left|\vec{x}-\vec{n}_{i}\right|}
\end{aligned}
$$

Thus the potential consist of $N-1$ punctures of $1 / r$ type, one of the punctures having been projected out to infinity. Note that the physical metric involves the (scale-setting) factor $\left(A_{1}\right)^{4}$. We can absorb it by introducing new, rescaled coordinates $\vec{y}=\left(A_{1}\right)^{2} \vec{x}$. The projected conformal factor takes now the form of

$$
\begin{aligned}
\tilde{\psi}(\vec{y}) & =1+\sum_{i=2}^{N} \frac{2 A_{i} A_{1} \sqrt{1+\frac{\left|\overrightarrow{\mathcal{N}}_{i}\right|^{2}}{4}}}{\left|\vec{y}-\overrightarrow{\mathcal{N}}_{i}\right|}= \\
& =1+\sum_{i=2}^{N} \frac{m_{i}}{2\left|\vec{y}-\overrightarrow{\mathcal{N}}_{i}\right|}
\end{aligned}
$$

with rescaled positions of the punctures $\overrightarrow{\mathcal{N}}_{i}=\left(A_{1}\right)^{2} \vec{n}_{i}$. The mass parameters of the punctures take the form of

$$
m_{i}=4 A_{i} A_{1} \sqrt{1+\frac{\left|\overrightarrow{\mathcal{N}}_{i}\right|^{2}}{4}} .
$$

They have the dimension of mass, but do not correspond exactly to the ADM mass of the individual punctures measured at their infinities. For the black hole at $\overline{\mathcal{N}}_{1}$, for instance, the ADM mass is equal to

$$
M_{1}^{\mathrm{ADM}}=\sum_{i=2}^{N} m_{i}=4 A_{1} \sum_{i=2}^{N} A_{i} \sqrt{1+\frac{\left|\overrightarrow{\mathcal{N}_{i}}\right|^{2}}{4}} .
$$

This can also be expressed in terms of the original solution $\psi$ :

$$
M_{1}^{\mathrm{ADM}}=4 A_{1} \sum_{i=1}^{N} A_{i} \sqrt{\frac{2}{1-\bar{N}_{i} \cdot \bar{N}_{1}}} .
$$

Analogous relations hold for other black holes.

If we project the 8-vertex solution 15 down to $\mathbf{R}^{3}$, it becomes an asymptoticallyflat configuration described by 22 with 7 punctures at points

$$
\begin{aligned}
& \overrightarrow{\mathcal{N}}_{2}=(0,0,0), \\
& \overrightarrow{\mathcal{N}}_{3}=(2,0,0), \\
& \overrightarrow{\mathcal{N}}_{4}=(-2,0,0), \\
& \overrightarrow{\mathcal{N}}_{5}=(0,2,0), \\
& \overrightarrow{\mathcal{N}}_{6}=(0,-2,0), \\
& \overrightarrow{\mathcal{N}}_{7}=(0,0,2), \\
& \overrightarrow{\mathcal{N}}_{8}=(0,0,-2) .
\end{aligned}
$$

and with mass parameters $m_{2}=4$ and $m_{3}, \ldots, m_{7}=4 \sqrt{2}$. The vertices, edges and marginally outer-trapped surfaces (MOTSs) projected to $\mathbf{R}^{3}$ are presented on Figure 2 . 

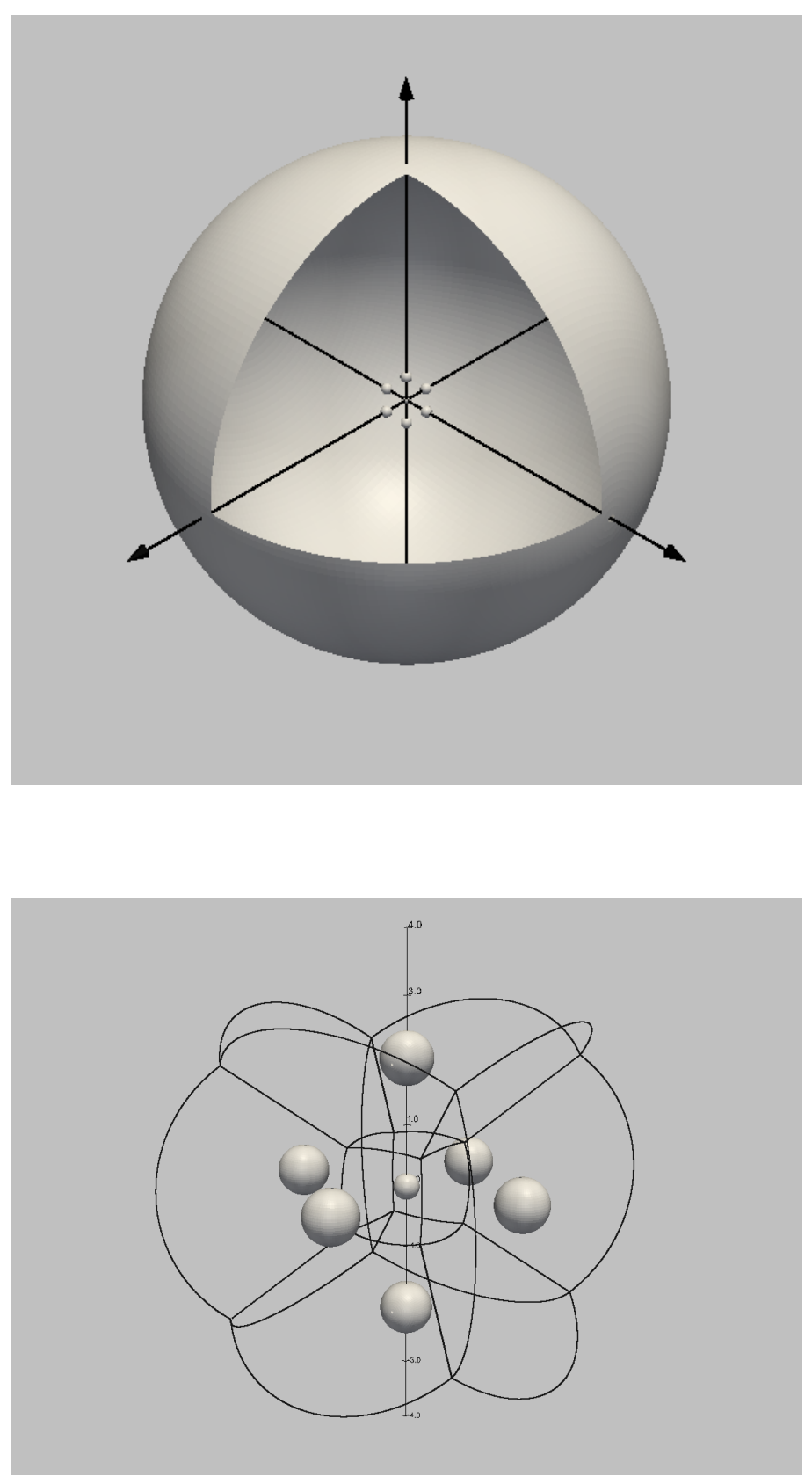

Figure 2. Initial MOTSs and elementary cells for the 8-black-hole configuration, projected to $\mathbf{R}^{3}$. The marginal surface corresponding to the black hole at infinity encompasses the whole configuration. Note that the 8 cubical lattice cells are isometric after the conformal rescaling. 


\section{Evolution}

We perform the three-dimensional evolution of this initial-data set using the Einstein Toolkit 23]; in particular, we use the McLachlan code [24, 25] to perform a finite-difference evolution of the Einstein's equation with adaptive-mesh-refinement capabilities provided by Carpet [26]. We have also made use of AHFinderDirect [27] to search for trapped surfaces.

Details of the evolution scheme can be found in Appendix A, the numerical error analysis is performed in Appendix B. We evolve the initial data presented in section 2.2 in a cubic box of side $40 M$ (here and in the following, we will adopt $M=m_{2} / 4$ as the unit of mass, length and time), with a spacing $\Delta_{0}=1 M$. We refine the grid at the seven black-hole locations using eight concentric grids for each location, down to a resolution of $\Delta_{8}=\Delta_{0} / 2^{8}=0.00390625 M$.

The eight initial MOTSs have been shown in Figure $2{ }^{+}$. Notice that, due to the stereographic projection, the large marginal surface initially at a radius of $20 M$ is inner trapped, rather than outer trapped, i.e. it is the expansion of the ingoing null normal that vanishes while the expansion of the outer normal is positive. It is therefore not a common apparent horizon of the sort usually encountered in binary mergers. This has an interesting side effect: the outer boundary conditions are causally disconnected from the region enclosed by this surface.

We find that the MOTSs at $( \pm 2,0,0),(0, \pm 2,0)$ and $(0,0, \pm 2)$ take approximately $130 M$ (in coordinate time) to merge to the MOTS at the origin, and approximately $170 M$ to merge to the larger, inner-trapped one, as illustrated in Figure 4 (the asymmetry here is due to the non-uniform numerical slicing). The evolution of the $z=0$ sections of the marginal surfaces are shown in Figure 3 , while the mean coordinate radii and masses for the the black holes initially at $(0,0,0),(2,2,2)$ and infinity are plotted in Figures 5 and 6

From the cosmological standpoint, we are particularly interested in the scaling of lengths with proper time. There are at least two candidate quantities for measuring the scaling of distances in this system: the (minimal) proper distance between nearneighbour surfaces and the proper length of each cell's edges. Note that these estimates need not agree with each other, since the expansion rate may well be different at different points.

In order to calculate proper lengths as functions of proper time, we restrict our attention to the $1+1$ subspace spanned by a reprentative curve in time, and obtain the proper time and the $x$-coordinate of gaussian observers with the following relations:

$$
\begin{aligned}
& \tau(t, x)=\int_{0}^{t} \alpha\left(t^{\prime}, x\right) \mathrm{d} t^{\prime} \\
& x_{\mathrm{g}}\left(t, x_{\text {init }}\right)=x_{\mathrm{g}}\left(t-\Delta t, x_{\text {init }}\right)-\int_{t-\Delta t}^{t} \beta^{x}\left(t^{\prime}, x_{\mathrm{g}}\left(t-\Delta t, x_{\text {init }}\right)\right) \mathrm{d} t^{\prime}
\end{aligned}
$$

where $x_{\text {init }}$ is the location of the observer at $t=0$.

The proper distance between marginal surfaces, as a function of proper time, is then given by:

$D(\tau)=\int_{\gamma_{\tau}}\left[\left(-\alpha^{2}(\tau, \ell)+\beta^{2}(\tau, \ell)\right)\left(\partial_{\ell} t\right)^{2}+\beta_{i}(\tau, \ell) \partial_{\ell} t \partial_{\ell} x^{i}+\gamma_{i j}(\tau, \ell) \partial_{\ell} x^{i} \partial_{\ell} x^{j}\right]^{1 / 2} \mathrm{~d} \ell$

+ We really only track the surfaces corresponding to the black holes at the origin, at infinity, and on the positive $x$-axis. The locations and shapes of the remaining five surfaces, included in the plots for clarity, are obtained by symmetry arguments. 

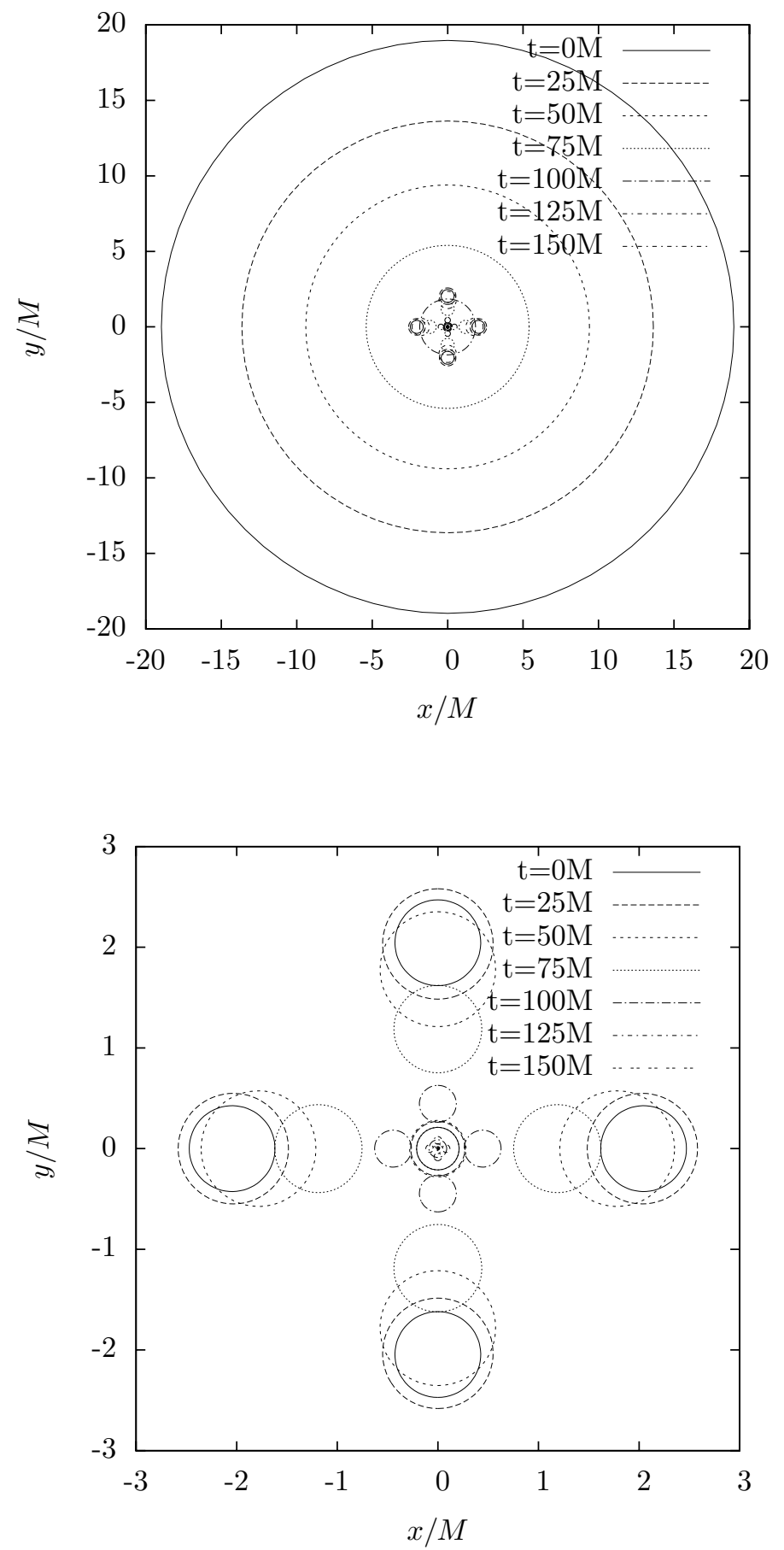

Figure 3. Section of the eight marginal surfaces on the $z=0$ plane, at times $t=(0,25,50,75,100,125,150) M$. The bottom plot is a zoomed-in version of the seven central MOTSs only. 

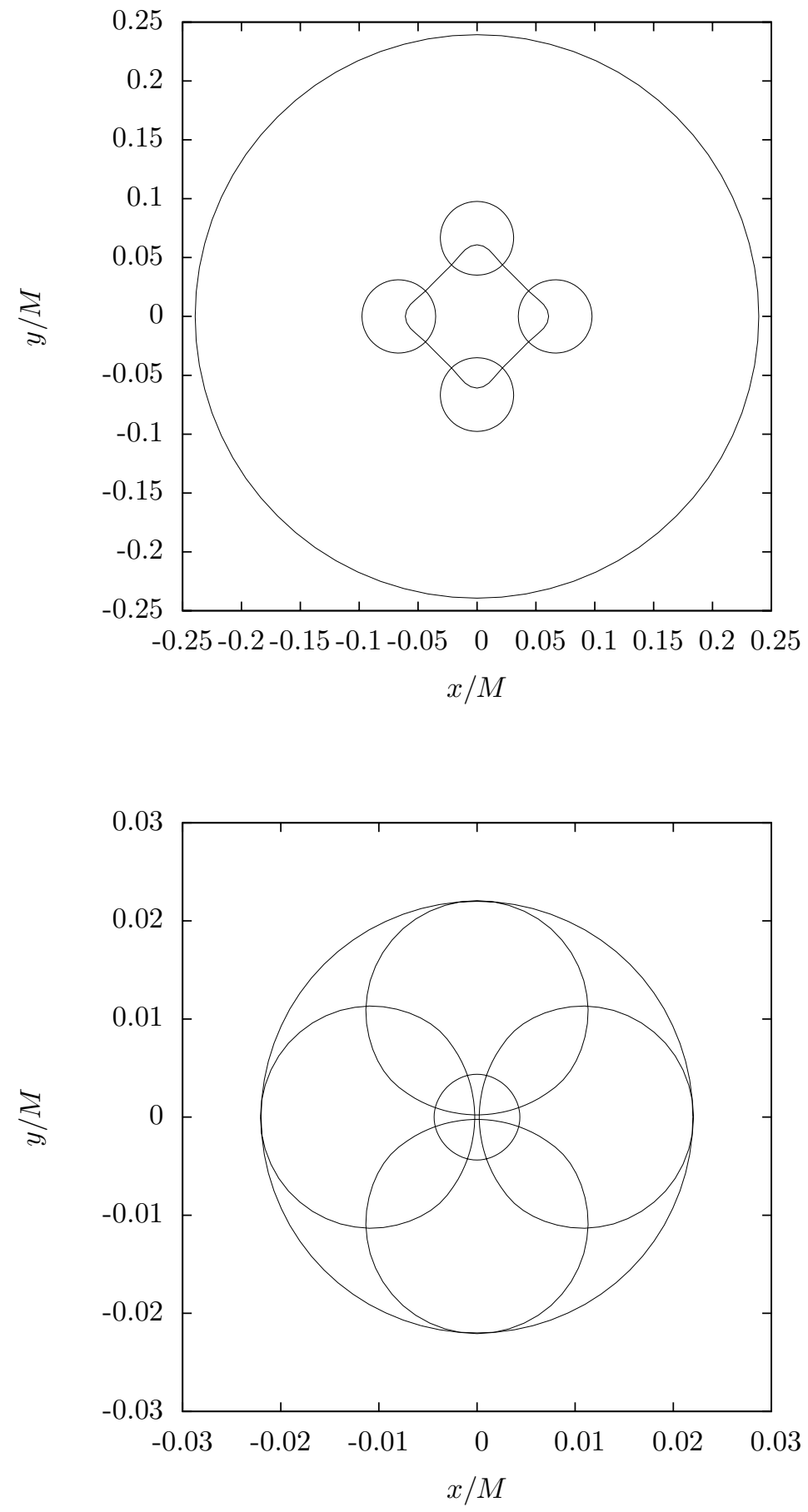

Figure 4. Section of the eight marginal surfaces on the $z=0$ plane, on the spatial slice of the first surface merger, corresponding to coordinate time $t=128 \mathrm{M}$ (top) and on that of the second merger at $t=167 M$ (bottom). 


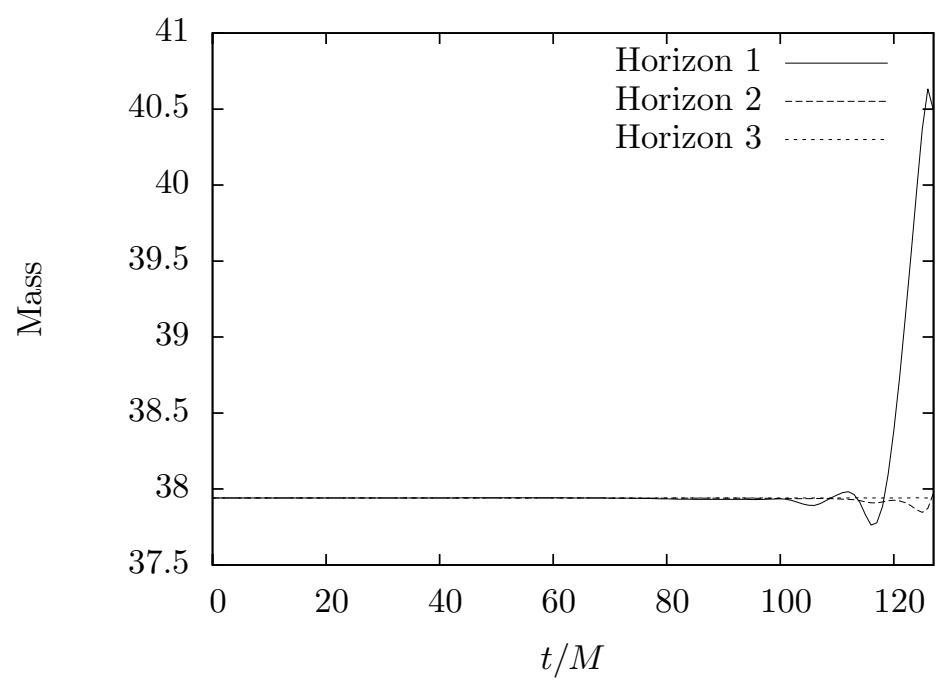

Figure 5. Masses of the three marginal surfaces of the black holes at $(0,0,0)$, $(2,2,2)$ and infinity, as a function of coordinate time.

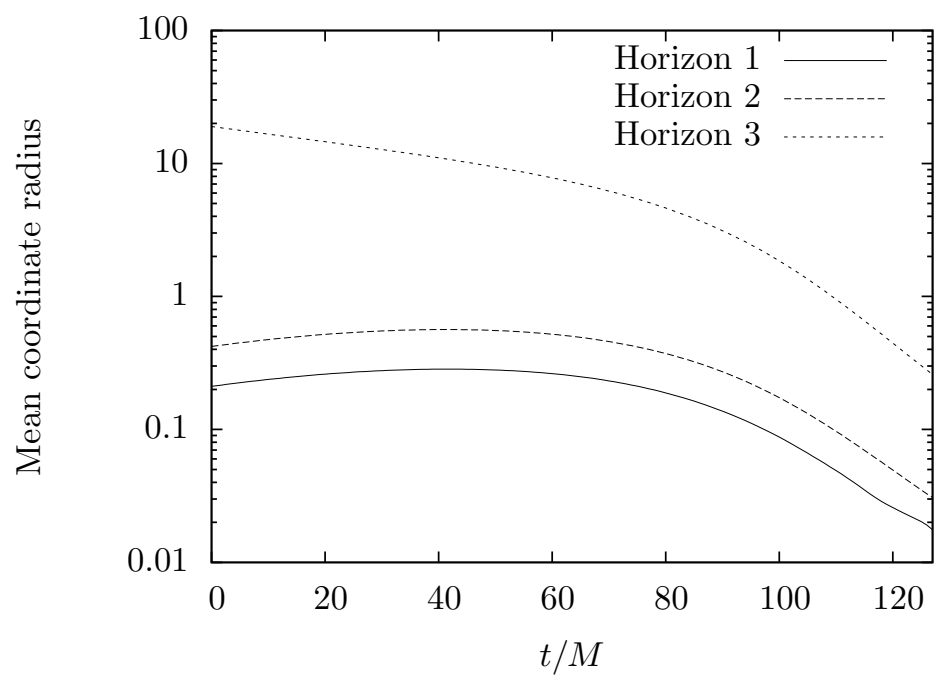

Figure 6. Mean coordinate radii of the three marginal surfaces of the black holes at $(0,0,0),(2,2,2)$ and infinity, as a function of coordinate time. 


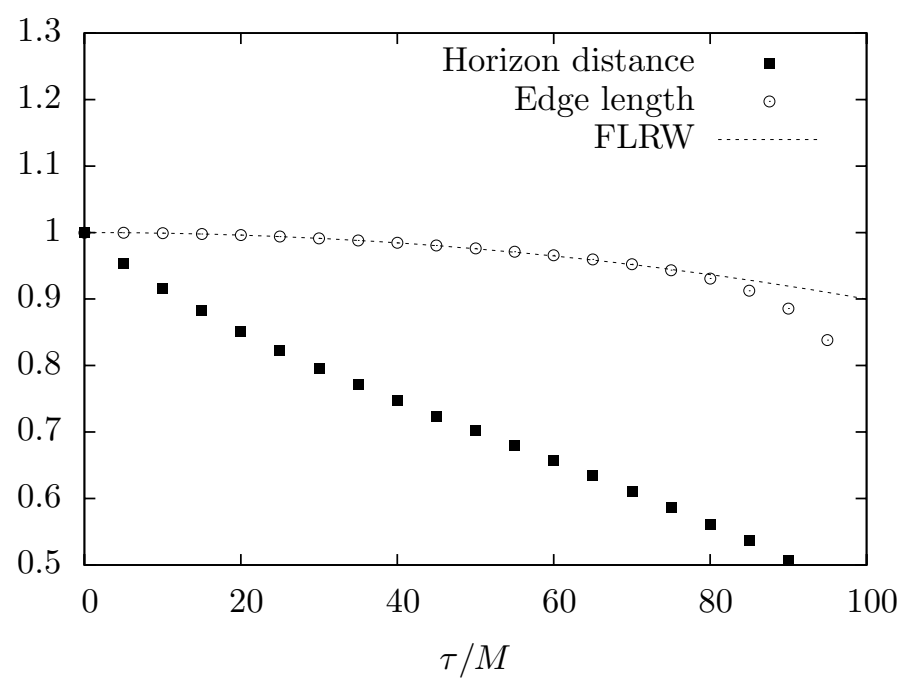

Figure 7. Several measures of scaling in the eight-black-hole universe, as functions of proper time $\tau$, plotted against a possible identification of the corresponding FLRW model (see section 4 for details). All the quantities have been renormalized to their respective values at $\tau=0$.

where $\gamma_{\tau}$ is the shortest constant- $\tau$ geodesic, parametrized by $\ell$, connecting two surfaces. We measure this quantity for the two outer-trapped surfaces initially at $(0,0,0)$ and $(2,0,0)$, the geodesic lying on the $x$-axis for symmetry reasons. This quantity is plotted in Figure 7 .

Similarly, the proper length of a lattice edge is given by equation (35), where now $\gamma_{\tau}$ is the constant- $\tau$ geodesic, parametrized by $\ell$, connecting the two vertices. It is easy to work out the initial shape of the cells, illustrated in Figure 2. The initial locations of the 16 vertices are given by $( \pm 2 / 3, \pm 2 / 3, \pm 2 / 3)$ and $( \pm 2, \pm 2, \pm 2)$. For simplicity, we choose to focus on the edge connecting $(2 / 3,2 / 3,2 / 3)$ to $(2,2,2)$, which, for symmetry reasons, always lies along the $x=y=z$ diagonal. The edge's proper length as a function of proper time is also shown in Figure 7 .

For reference, the relative spatial and temporal scales of the system are illustrated in Figures 8 and 9 . The numerical locations of the two vertices during the evolution is shown in Figure 8 , along with a few other representative points on the geodesic and the constant- $\tau$ lines. In Figure 9, we also show the span of the numerical coordinates of the cell edge in $\left(\tau, x_{\mathrm{g}}\right)$ space: this illustrates how the gauge condition adopted in this simulation freezes the evolution around $\tau \lesssim 150 M$, preventing us from observing the system's behavior after this time. In Figure 8, we also plot the intersection of the marginal surfaces surrounding the black holes at the origin and at infinity with the $x=y=z$ diagonal; this illustrates that, after $t \sim 120 M$, the inner vertex is quite close to the MOTS of the black hole at the origin: by this time, we can expect finite-size effects to play a significant role in the scaling of lengths. Additionally, we 


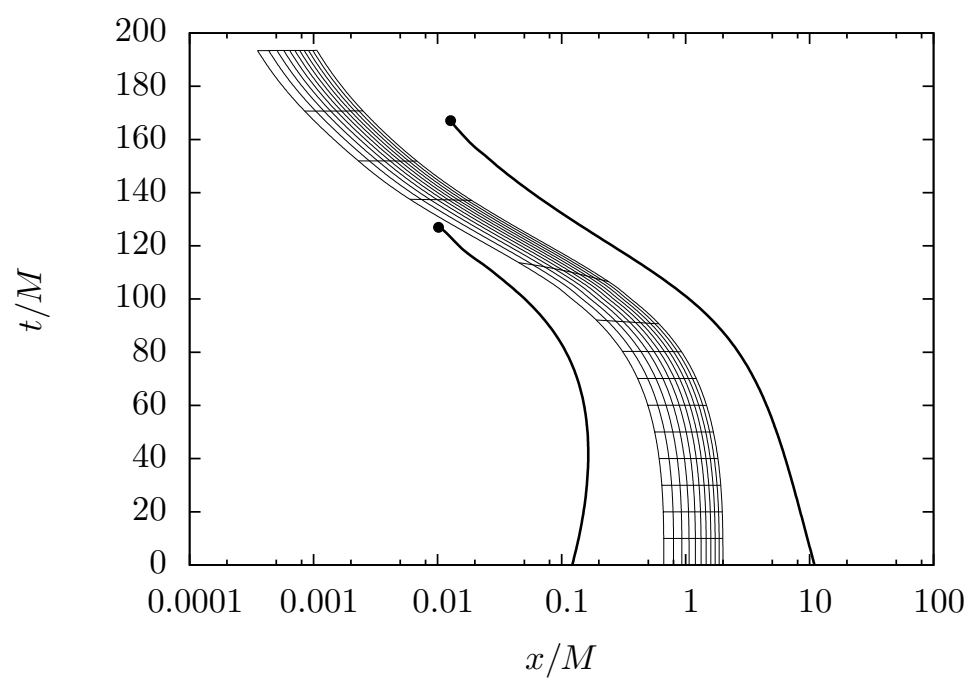

Figure 8. Coordinate $x_{\mathrm{g}}$ of gaussian observers and constant- $\tau$ lines $(\tau=$ $0,10, \ldots, 140 M)$ on the $x=y=z$ diagonal, for points initially located between the two vertices at $(2 / 3,2 / 3,2 / 3)$ and $(2,2,2)$. The thick lines represent the $x$ coordinate of the intersections of the marginal surfaces at $(0,0,0)$ (left) and at infinity (right) with the diagonal, up until the mergers at $t=128 \mathrm{M}$ and $t=167 \mathrm{M}$, respectively.

show in Appendix B that the numerical error quickly degrades after $\tau \sim 80 M$. Based on these considerations, we only show the scaling up to proper times of about $100 \mathrm{M}$ in Figure 7

Figure 7 also includes a possible counterparts of the eight-black-hole lattice in the FLRW class: one with the same initial edge length. This definition is made more precise in section 4 , where we will constrast these results with the large-scale dynamics of a perfectly homogeneous and isotropic universe.

Finally, let us notice here in passing that the interest of this toy model goes beyond the cosmological application. In particular, it provides an interesting example of overlapping MOTSs within the framework of a BSSN evolution.

\section{Comparison with the FLRW class}

The comparison of the configuration with an FLRW model requires solving Ellis' "fitting problem" 5, i.e. determining the parameters characterizing the reference FLRW model which our configuration resembles most closely. There are infinitely many ways to do this; we will sketch below the procedure we will use in this paper based on the quantities measured in section 3 .

Due to the symmetry group of our configuration, the reference FLRW model is a closed model $(k=1)$, with spatial slices of spherical topology. The matter content is represented by dust. Since the primary variable describing any FLRW model is the scale factor, representing the scaling of lengths in time, the first step in the comparison 


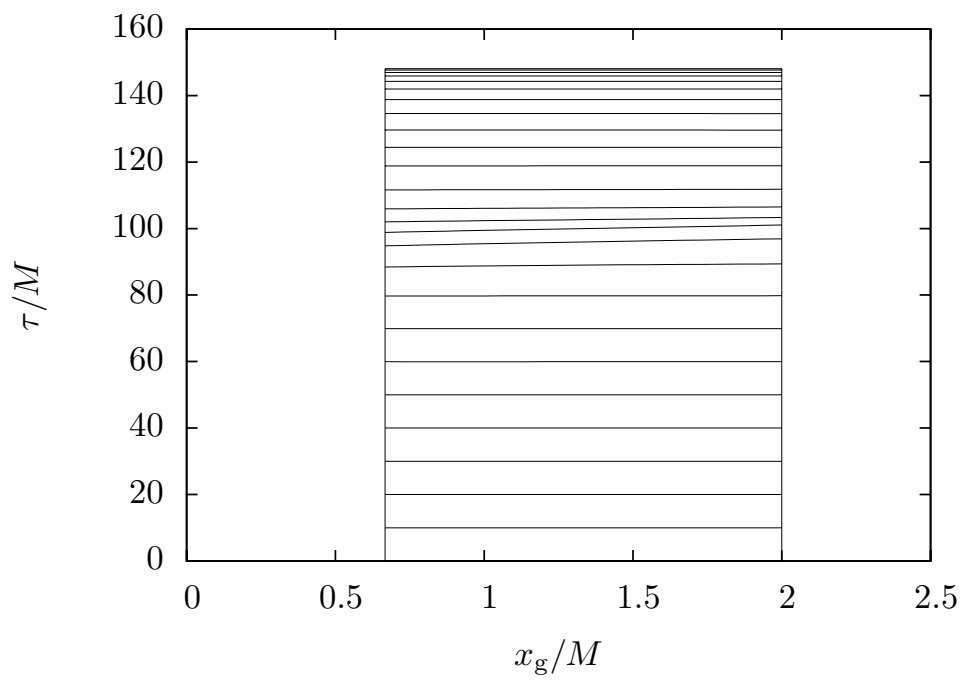

Figure 9. Space-time region spanned by the edge between $(2 / 3,2 / 3,2 / 3)$ and $(2,2,2)$, represented in $\left(\tau, x_{\mathrm{g}}\right)$ coordinates. The slicing adopted in this work only extends, in this region, up to $\tau \lesssim 150 M$. The horizontal lines are constant- $t$ lines, i.e. sections of the spatial hypersurfaces used in the simulation.

is to identify some measure of length in the lattice universe. We required this variable to be non-local, thereby capturing the large-scale, average behaviour of the universe rather than the local physics at a single, arbitrarily chosen point. The total volume of the configuration, which is the most obvious parameter for a closed universe, is obviously infinite and therefore of no use for our purpose.

The problem of the size measurement is a bit simplified by the discrete symmetry of the model. In section 3 , it seemed reasonable to choose the variable in a way which is consistent with the cell structure of the black hole lattice. The first obvious choice would be to use the geodesic distance $D_{\text {hor }}(\tau)$ between the MOTSs of two neighbouring black holes. Recall that a MOTS is a closed two-surface whose null expansion vanishes in one direction 28. We have observed that the behavior of this quantity for small times varies considerably from the behavior of the size parameter of a closed FLRW (see Figure 7). In particular, the derivative of $D_{\text {hor }}(\tau)$ does not vanish at $\tau=0$, but rather approaches the limiting value $\dot{D}_{\text {hor }}(0)=-2$, despite the configuration being momentarily static and symmetric with respect to time reflections. This striking phenomenon can be explained by the fact that the black hole MOTSs at $\tau=0$ are all bifurcation surfaces of the horizon. One can check that in our coordinates the corresponding MOTSs seem to approach each other at approximately the speed of light even at the moment of maximal expansion (see Appendix C). This makes $D_{\text {hor }}(\tau)$ an unsuitable size parameter for the purpose of FLRW fitting.

Our second choice for the size variable was the geodesic length of the individual cell's edge, i.e. the geodesic distance between the two vertices of an individual cube, also used in [22]. The edges lie relatively far from the punctures, and thus from the 
potentially problematic black hole region, at all times. Hence we can hope they are resolved quite well during the simulation.

The mapping to the FLRW class is then carried out by fitting the size $a_{\mathrm{eff}}(t)$ of the reference sphere discussed in section 2.1 by demanding that the length of the edge match exactly the length of the corresponding edge of the cubical tiling of a round (FLRW) sphere. At the same time this fitting gives the effective Ricci curvature ${ }^{(3)} R_{\text {eff }}$ as the curvature of the corresponding FLRW sphere:

$$
{ }^{(3)} R_{\mathrm{eff}}=\frac{6}{a_{\mathrm{eff}}^{2}} .
$$

At first sight, it may seem quite strange that the effective Ricci curvature is not given by any kind of average, over a domain in the constant time slice, of the local values of the curvature. Keep in mind however that in the FLRW class, due to the high symmetry, the value of the (constant) Ricci curvature is directly related to infinitely many other parameters characterizing the geometry, for example the relation between the volume and the area of spheres, the angle deficits, the geodesic focusing at any point, and so on. Since the geometry of the discrete models is not homogeneous, these simple relations are lost. Nevertheless it is not a priori clear which of those parameters we should regard as a convenient inhomogeneous generalization of the spatial ${ }^{(3)} R$ appearing in the Friedmann equation. The definition of ${ }^{(3)} R_{\text {eff }}$ we propose here is based on rescaling the value of the Ricci curvature of a unit 3-sphere by the ratio of the size of the lattice edges. As we shall see, it provides, together with a complementary definition of the effective energy (see below), an excellent fit for the dynamics of the inhomogeneous lattice until up the time of $t \approx 80$, when we start losing resolution (mostly due to an outgoing shift vector that makes the system rapidly shrink in coordinate size). Independently from this limit, after $\tau \sim 100 M$ the eight MOTSs rapidly swallow up the whole spatial slices, making this model less and less appropriate to describe a universe filled with point-like masses.

In order to derive an effective energy density, let us recall that the configuration is at rest at $t=0$, so we can assume that $\dot{a}_{\text {eff }}(0)=0$ and use Friedmann's equation to obtain $\rho_{\text {eff }}$

$$
0=\frac{8 \pi}{3} \rho_{\text {eff }}-\frac{{ }^{(3)} R_{\text {eff }}}{6} .
$$

It is instructive to compare the effective total mass, obtained as the product of $\rho_{\text {eff }}$ with the volume of the FLRW sphere, with the total matter content of our configuration:

$$
M_{\text {eff }}=\rho_{\text {eff }} 2 \pi^{2} a_{\text {eff }}^{3}=378.78, \quad M_{8 \mathrm{BH}}=8 M_{\mathrm{ADM}}=303.53
$$

Clearly the effective mass is around 25\% larger than the sum of ADM masses of the individual black holes. This is consistent with the expected nonlinear effects of gravitation, as the strong gravitational fields gravitate themselves. It also agrees quite well with [22, where the fit with the corresponding FLRW model is based on imposing the equality of the total masses and the lengths of the edges are used for comparison instead.

\section{Conclusions}

We have discussed the construction of the initial data corresponding to periodic lattices of black holes using the Lichnerowicz-York construction. We have shown that just like in FLRW class there is a link between, on one hand, the curvature of the underlying 
constant-curvature metric (and thus also the topology of the spatial slice) and, on the other hand, the matter content and the Hubble parameter. In particular, it follows that it is not possible to find a flat, rectangular lattice of black holes without a momentary expansion or contraction.

We then recalled the construction of periodic lattices of black holes on an $S^{3}$ sphere with vanishing extrinsic curvature, originally introduced in [21]. We focused on the symmetric eight-black-hole configuration and showed that it can be conformally projected to an asymptotically-flat seven-black-hole configuration with unequal mass parameters. We have discussed briefly its basic properties and then showed the results of the numerical evolution of this system. The main goal was the comparison with the time evolution of a dust-filled closed FLRW model, evolved from the maximal expansion moment until the recollapse.

We proposed two methods for measuring the effective size of the configuration: the geodesic distance between the MOTSs of two neighbouring black holes and the geodesic length of the edge of a single cell. The first one turned out to behave very differently from the size parameter of FLRW, as its derivative did not seem to vanish even at the initial slice. We explained this unusual behavior by noting that, at the maximum expansion, each MOTS is a bifurcation surface where two distinct marginal tubes intersect. The edge length, on the other hand, calculated in normal coordinates and in proper time, seems to follow very closely the evolution of a closed FLRW if we fit its size (and consequently its curvature and mass) in an appropriate way. Despite our configuration being very far from homogeneity, the effective size obeys the Friedmann equation to a remarkable degree up to times of $t \approx 80 M$, which is approximately $30 \%$ of the recollapse time of the FLRW. After that time our simulation is simply unable to resolve the system. The only observable backreaction (or coarse-graining) effect in our simulation seems to lie in the effective total mass of the system, which turns out to be $25 \%$ larger that the sum of masses of the individual black holes. In other words, the eight-black-hole lattice does mimic a closed FLRW dust model, but one whose total mass is substantially larger than that due to the black holes alone.

It is of course not clear to what extent these results will hold if we consider other types of models (flat or open, with a positive initial expansion) or if we drop the assumption of existence of a large group of discrete symmetries. Note that at first sight the symmetry assumption may look like an innocent ansatz whose only purpose is to simplify the geometry of the problem. Nevertheless it is important to understand that it is in fact quite restrictive. In particular it prohibits many types of interactions between the matter inhomogeneities, such as two-black-hole mergers or interactions via low- $\ell$ spherical harmonic modes. Configurations which do not have that kind of symmetry may potentially exhibit many other effects of backreaction.

\section{Acknowledgements}

We would like to thank Lars Andersson and Niall Ó Murchadha for useful discussions and comments. We also acknowledge the Erwin Schrödinger Institute in Vienna for its support and hospitality during the 2011 "Dynamics of General Relativity: Numerical and Analytical Approaches" programme, where this work was started. M.K. acknowledges hospitality by the Max-Planck-Institut für Gravitationsphysik. E.B. is funded by a Marie Curie International Reintegration Grant PIRG05-GA-2009249290. Computations were carried out on the MPI-GP Damiana and Datura clusters. 


\section{Appendix A. Evolution system}

In order to solve Einstein's equation, we use the McLachlan code, which implements a finite-difference discretization of the BSSN formulation [29, 30, 31].

$$
\begin{aligned}
\left(\partial_{t}-\beta^{l} \partial_{l}\right) W= & -\frac{1}{3} \alpha K+\frac{1}{3} \partial_{i} \beta^{i} \\
\left(\partial_{t}-\beta^{l} \partial_{l}\right) K= & -D_{i} D^{i} \alpha+\alpha\left(\bar{A}_{i j} \bar{A}^{i j}+\frac{1}{3} K^{2}\right) \\
\left(\partial_{t}-\beta^{l} \partial_{l}\right) \bar{\gamma}_{i j}= & -2 \alpha \bar{A}_{i j}+2 \bar{\gamma}_{i(j} \partial_{k)} \beta^{i}-\frac{2}{3} \bar{\gamma}_{i j} \partial_{k} \beta^{k} \\
\left(\partial_{t}-\beta^{l} \partial_{l}\right) \bar{A}_{i j}= & W^{2}\left(-D_{i} D_{j} \alpha+a R_{i j}\right)^{T F} \\
& +\alpha\left(K \bar{A}_{i j}-2 \bar{A}_{i k} \bar{A}_{j}^{k}\right) \\
& +2 \bar{A}_{k(i} \partial_{j} \beta^{k}-\frac{2}{3} A_{i j} \partial_{k} \beta^{k} \\
\left(\partial_{t}-\beta^{l} \partial_{l}\right) \bar{\Gamma}^{i}= & \bar{\gamma}^{j k} \beta^{i} \partial_{j} \beta_{k}+\frac{1}{3} \bar{\gamma}^{i j} \partial_{j} \partial_{k} \beta_{k}-\bar{\Gamma}^{j} \partial_{j} \beta^{i} \\
& +\frac{2}{3} \bar{\Gamma}^{i} \partial_{j} \beta^{j}-2 \bar{A}^{i j} \partial_{j} \alpha \\
& +2 \alpha\left(\bar{\Gamma}_{j k}^{i} \bar{A}^{j k}-3 \bar{A}^{j k} \partial_{k} \ln W-\frac{2}{3} \bar{\gamma}^{i j} \partial_{j} K\right)
\end{aligned}
$$

where $\gamma_{i j}=W^{-2} \bar{\gamma}_{i j}$ is the three-metric, $K_{i j}=\frac{K}{3} \gamma_{i j}+W^{-2} \bar{A}_{i j}$ is the extrinsic curvature, and $W=\operatorname{det} \gamma^{-1 / 6}$ and $\bar{\Gamma}^{i}=-\partial_{j} \bar{\gamma}^{i j}$ are auxiliary variables. The gauge variables are evolved according to:

$$
\begin{aligned}
& \left(\partial_{t}-\beta^{i} \partial_{i}\right) \alpha=-2 \alpha K \\
& \left(\partial_{t}-\beta^{i} \partial_{i}\right) \beta^{j}=\frac{3}{4} B^{j} \\
& \left(\partial_{t}-\beta^{i} \partial_{i}\right) B^{j}=-\left(\partial_{t}-\beta^{i} \partial_{i}\right) \bar{\Gamma}^{j}-\eta B^{j}
\end{aligned}
$$

with $\eta=1$.

We use fourth-order finite-differencing. An important difference with standard black-hole evolutions is that we choose $\alpha=1$ everywhere as the initial condition, because a precollapsed lapse $\alpha=\tilde{\psi}^{-2}$ (see $(21)$ ) leads to a vast collapsed region at the center of the domain, which unnecessarily slows down the proper-time evolution of the black holes.

\section{Appendix B. Numerical error on the proper distance estimate}

Estimating the numerical error associated with a three-dimensional, complex simulation is notoriously difficult. Additionally, our scheme for computing the proper distance does not only involve finite differencing and AMR operations, but also a number of post-processing steps, in particular reslicing $1+1$ spaces in terms of gaussian observers (which involves using (33) and (34) to obtain the gaussian coordinates, and then an interpolation in both time and space) and integrating the line element to obtain a proper distance.

In order to quantify the cumulative error of this procedure, we evolve the same initial data at two additional resolutions, corresponding to a spacing of $\Delta_{0}=2 M$ and $\Delta=4 M$ on the coarsest grid, and compare the edge proper length to obtain an order of magnitude and a scaling for the numerical error on this quantity. The result 


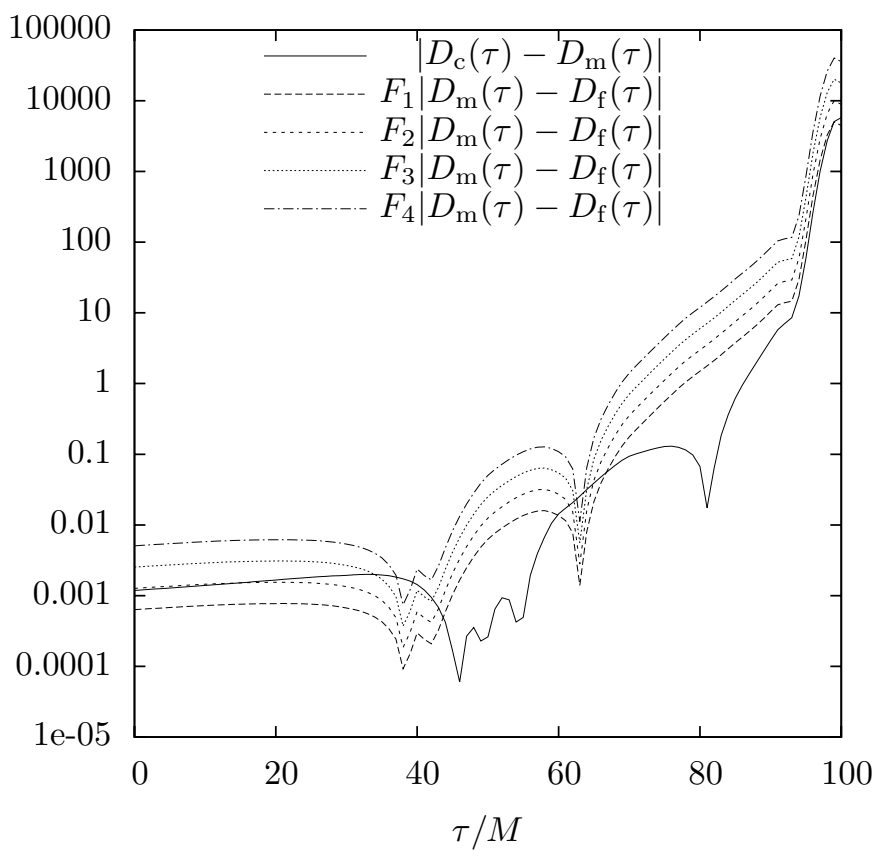

Figure B1. Convergence study for the edge proper length. $D_{\mathrm{c}}(\tau), D_{\mathrm{m}}(\tau)$, and $D_{\mathrm{f}}(\tau)$ are the edge proper lengths for the coarse, medium and fine resolution respectively. The factors $F_{i}$ represent $i$-th order scaling of the error with the lattice spacing.

is shown in Figure B1 the error scales like the second power of the lattice spacing up until $t \sim 40 M$, degrading afterwards. The order of magnitude is below $1 M$ by $t=80 M$ (or about $0.6 \%$ of the proper distance); this seems like a reasonable number to represent the numerics-related error bar on the proper distance.

\section{Appendix C. Geodesic distance between the MOTSs}

In this appendix we will argue that the minimal surfaces around the punctures at $t=0$ are all bifurcation surfaces, i.e. they give rise to two MOTTs, one propagating towards the asymptotically flat end of the manifold and the other in the other direction. Let $\Sigma_{0}$ be the $t=0$ hypersurface. Consider the evolution of the initial data near $t=0$ with gauge conditions $\alpha=1$ and $\beta^{i}=0$ at $\Sigma_{0}$, as we have chosen on our initial hypersurface for the numerical evolution.

We define two null normals to $D$ given by $l=\frac{\sqrt{2}}{2}(T+R)$ and $k=\frac{\sqrt{2}}{2}(T-R)$, see Figure C1. It is easy to see that the expansion of both null normals $\theta^{l}$ and $\theta^{k}$ vanishes. The reason for that is the fact that $D$ is minimal, therefore the expansion with respect to $R$ vanishes, and that the initial data is time-symmetric $\left(K_{i j}=0\right)$, so the expansion in $T$ direction is zero as well.

The behavior of the MOTS is determined by the continuation equation for a 


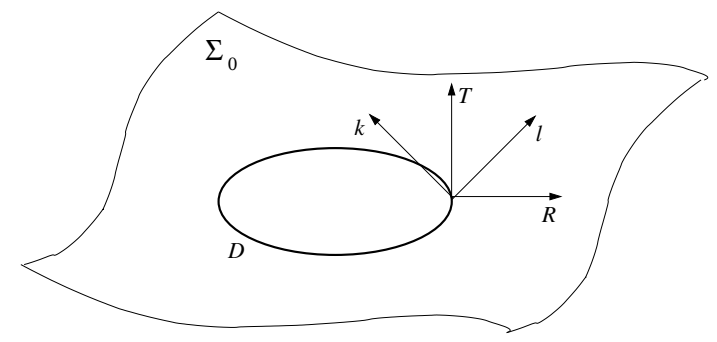

Figure C1. Minimal surface $D$ as a subsurface of $\Sigma_{0}$ and its normals.

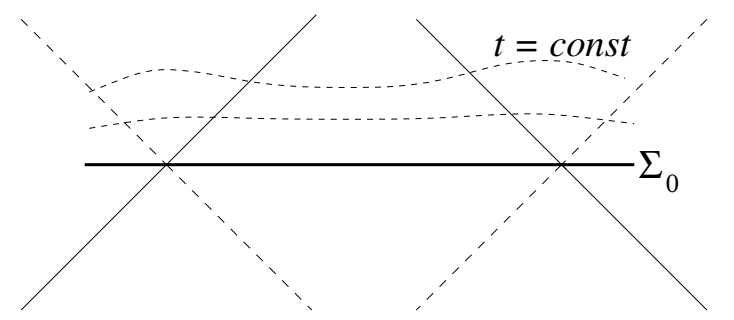

Figure C2. Two neighbouring minimal surfaces at $t=0$ as bifurcation surfaces. The outward-moving MOTTs (solid line) seem to expand with approximately light speed.

MOTT, which gives the condition under which a variation of a MOTS preserves the vanishing of one of the null expansions, see [32, 28]. Let now $n=n_{l} l+n_{k} k$ be the variation vector field. Since both null expansions vanish, we may continue the MOTS by imposing either the condition $\theta^{l}=0$ or $\theta^{k}=0$. In the first case the continuation equation reads

$$
\left(\Delta-2 \omega^{A} D_{A}-\left(\frac{\mathcal{R}}{2}+D_{A} \omega^{A}-\omega_{A} \omega^{A}\right)\right) n_{k}-n_{l}\left|\sigma_{l}\right|^{2}=0 .
$$

$\Delta$ denotes here the Laplace operator on $D, \omega_{A}=\nabla_{A} k^{a} l_{a}, \sigma_{l}$ is the shear of $l$ and $\mathcal{R}$ is the scalar curvature of $D$.

It turns out that both the surface $D$ itself and the physical 3-metric $\gamma$ in its vicinity are almost exactly spherically symmetric. The reason is that $D$ lies relatively close to the pucture $p$, at radius $r \approx 0.21$ in the $\mathbf{R}^{3}$ variables, small comparing to the distance of 2 between the punctures. The round 3 -sphere metric $\gamma^{S}$ is of course spherically symmetric around the puncture. The conformal factor $\psi$ is dominated in this region by the singularity at the origin and the sum of six terms coming from other punctures. The latter is not exactly spherically symmetric, but the non-spherical contributions from those 6 terms cancel out to a great degree due to their symmetric alignment. Therefore the relative variation of $\psi$ in angular variables at that place is only of the order of $10^{-5}$. All deviations from spherical symmetry are thus of very small order of magnitude, so in the first approximation we may neglect all terms in C.1 which break the spherical symmetry of $\gamma$, i.e. those containing the vector field 


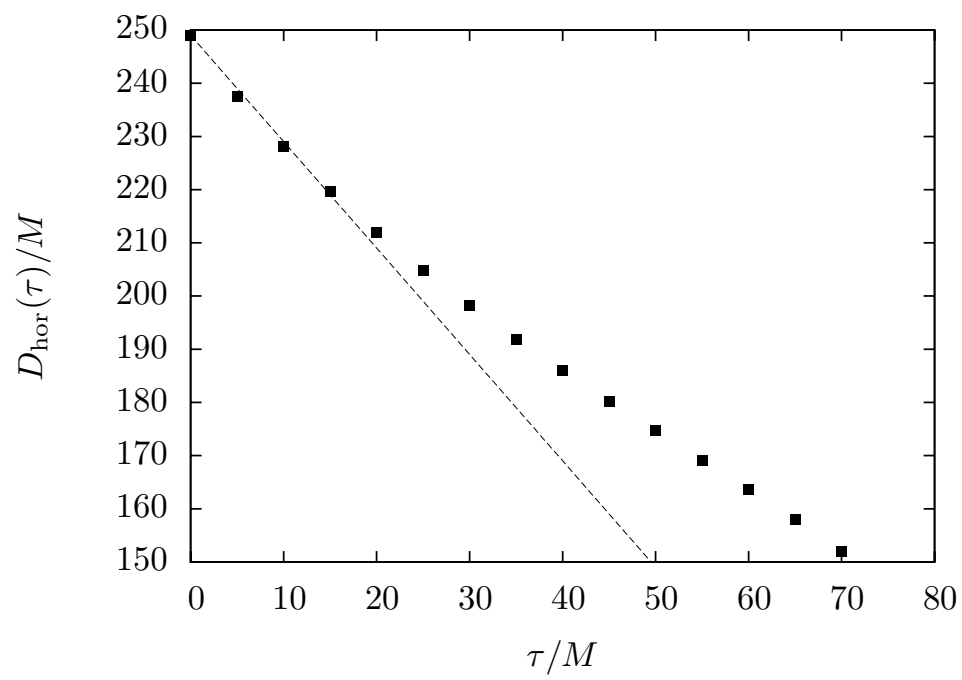

Figure C3. The proper distance between near-neighbour MOTSs as a function of proper time. The straight line has a slope of -2 .

$\omega$ and the tensor field $\sigma_{l}{ }^{*}$. The resulting equation now reads

$$
\left(\Delta-\frac{\mathcal{R}}{2}\right) n_{k}=0 \text {. }
$$

The solution is straightforward: $n_{k}=0, n_{l}$ being an arbitrary function. This obviously corresponds to the continuation of the MOTT along the null vector field $l$. The same argument shows that we may continue the other MOTT along $k$. Both MOTTs are thus in very good approximation isolated horizons.

It is now clear why the derivative of geodesic distance between MOTSs does not vanish despite the configuration being time reflection symmetric. The MOTTs of two neighbouring black holes, when viewed in either Gaussian or numerical coordinates, seem to expand almost exactly at the light speed (equal to 1 in simulation units). This by no means contradicts the time reflection symmetry of the inital data, as the MOTT expanding in the future direction is accompanied by another one which expands in the past direction, see Figure C2. Figure C3, showing the distance between MOTSs along with the expected initial slope of -2 , provides support to the analysis above.

\section{References}

[1] Cervantes-Cota J L and Smoot G 2011 (Preprint 1107.1789)

[2] Sanders R 2010 The Dark Matter Problem: A Historical Perspective (Cambridge University Press) ISBN 9780521113014

[3] Wang Y 2010 Dark Energy (Wiley-VCH) ISBN 9783527409419

[4] Amendola L and Tsujikawa S 2010 Dark Energy: Theory and Observations (Cambridge University Press) ISBN 9780521516006

\footnotetext{
* Actually the shear term $\left|\sigma_{l}\right|^{2}$ in C.1, being quadratic, is of even lower order.
} 
[5] Ellis G and Stoeger W 1987 Class. Quantum Grav. 4 1697-1729

[6] Andersson L and Coley A 2011 Class. Quantum Grav. 28160301

[7] Korzyński M 2010 Class. Quantum Grav. 27105015

[8] Bolejko K, Célérier M N and Krasiński A 2011 Class. Quantum Grav. 28164002

[9] Buchert T 2011 Class. Quantum Grav. 28164007

[10] Clifton T 2011 Class. Quantum Grav. 28164011

[11] Brannlund J, Hoogen R v d and Coley A 2010 Int.J.Mod.Phys. D19 1915-1923

[12] Wiltshire D L 2009 Phys. Rev. D 80123512

[13] Wiltshire D L 2007 in "Dark Matter in Astroparticle and Particle Physics: Proceedings of the 6th International Heidelberg Conference", eds H.V. Klapdor-Kleingrothaus and G.F. Lewis, (World Scientific, Singapore, 2008) pp 565-596

[14] Clarkson C, Ellis G, Larena J and Obinna U 2011 Rep. Prog. Phys. 74112901

[15] Wiltshire D L 2011 Class. Quantum Grav. 28:164006, 2011

[16] Lindquist R W and Wheeler J A 1957 Rev. Mod. Phys. 29 432-443

[17] Clifton T and Ferreira P G 2009 Phys. Rev. D 80103503

[18] Pfeiffer H P 2012 (Preprint 1203.5166)

[19] Lichnerowicz A 1944 J. Math. Pures Appl. 23

[20] York James W J 1971 Phys. Rev. Lett. 26 1656-1658

[21] Wheeler J 1983 Foundations of Physics 13(1) 161-173 ISSN 0015-9018 10.1007/BF01889418

[22] Clifton T, Rosquist K and Tavakol R 2012 (Preprint 1203.6478)

[23] Loffler F, Faber J, Bentivegna E, Bode T, Diener P et al. 2011 (Preprint 1111.3344)

[24] Mclachlan code URL http://www.cct.lsu.edu/ eschnett/McLachlan/

[25] Kranc code URL http://kranccode.org/

[26] Schnetter E, Hawley S H and Hawke I 2004 Class. Quantum Grav. 21 1465-1488

[27] Thornburg J 2004 Class. Quantum Grav. 21 743-766

[28] Andersson L, Mars M and Simon W 2007 (Preprint 0704.2889)

[29] Nakamura T, Oohara K and Kojima Y 1987 Prog. Theor. Phys. Suppl. 90 1-218

[30] Shibata M and Nakamura T 1995 Phys. Rev. D 52 5428-5444

[31] Baumgarte T W and Shapiro S L 1999 Phys. Rev. D 59024007

[32] Korzyński M 2006 Phys. Rev. D 74104029 